\title{
El uso de armas químicas en Siria, un desafío para el derecho internacional
}

Fecha de recepción: 8 de noviembre de 2013

Fecha de aceptación: 25 de octubre de 2014

Doi: http://dx.doi.org/10.12804/acdi8.1.2015.01

\section{Rubén Cardoza Zúñiga*}

Resumen: Este artículo analiza las acciones tomadas por la comunidad internacional, a través de la Organización para la Prohibición de las Armas Químicas (OPAQ) y del Consejo de Seguridad sobre la amenaza que representa el hecho de poseer y, sobre todo, de usar armas químicas en contra de la población, como una reacción enérgica por parte del gobierno de Siria, violando las normas y principios más elementales del derecho internacional y del derecho internacional humanitario. Hay que reconocer que en esta ocasión se pudo lograr un consenso unánime entre los miembros del Consejo de Seguridad para imponer un proceso de destrucción de las armas químicas, materiales y aditamentos que posee el gobierno de la República de Siria, mediante la Resolución del Consejo de Seguridad 2118, sin tener que recurrir a las sanciones mencionadas en el capítulo VII de la Carta de las Naciones Unidas, por lo que imperó el derecho internacional. Como podrá observar el lector, las acciones en conjunto tomadas tanto en el seno del Consejo de Seguridad,

* Profesor investigador en Egap del Tecnológico de Monterrey en el área de Derecho Internacional Público. Posdoctorado en EGAP; doctor en Derecho por la Universidad Autónoma de Nuevo León. Investigador invitado en el Instituto Max Planck de Derecho Público Comparado y Derecho Internacional; profesor invitado en la Universidad Autónoma de Nuevo León.Correos electrónicos: rcardoza@itesm.mx, ruben_cardoza@hotmail.com. Dirección postal: EgAP Gobierno y Política Pública, Av. Eugenio Garza Lagüera y Rufino Tamayo s/n 66269, San Pedro Garza García, NL México.

Para citar este artículo: Cardoza Zúñiga, Rubén, "El uso de armas químicas en Siria, un desafío para el derecho internacional”, ACDI - Anuario Colombiano de Derecho Internacional, 2015, 8, pp. 17-40. Doi: http://dx.doi. org/10.12804/acdi8.1.2015.01 
por la OPAQ, como en el consenso llevado a cabo entre Rusia y los Estados Unidos obligaron al gobierno de Siria a comprometerse a aceptar lo estipulado en la Convención sobre Armas Químicas, y se evitó de esta manera que se realizara una invasión militar a Siria, situación que empezaba a ser considerada por el gobierno de los Estados Unidos.

Palabras clave: Siria, armas químicas, Consejo de Seguridad, Organización para la Prohibición de las Armas Químicas, Convención sobre las Armas Químicas.

\section{The Use of Chemical Weapons in Syria, a Challenge for the International Law}

Abstract: This article discusses actions taken by the international community, through the Organisation for the Prohibition of Chemical Weapons (OPCW) and the Security Council, about the threat that represents the fact that a Statelike Syria possess and used chemical weapons against its population as a violent reaction of the government of Syria, violating the most fundamental rules and principles of international law and international humanitarian law. However, we must recognize that on this occasion it was able to achieve a unanimous consensus among the members of the Security Council to impose a process of destruction of chemical weapons, materials and accessories that has the government of the Republic of Syria, through the Security Council Resolution 2118, avoiding to resort to the sanctions referred in the chapter VII of the United Nations Charter, prevailed by this way the international law and keep a military invasion off in Syria, a situation that was considered by the government of the United States.

Key words: Syria, chemical weapons, Security Council, Organisation for the Prohibition of Chemical Weapons, Chemical Weapons Convention.

\section{O uso de armas químicas na Síria, um desafio para o direito internacional}

Resumo: Este artigo analisa as ações tomadas pela comunidade internacional, através da Organização para a Proibição das Armas Químicas (OPAQ) e do Conselho de Segurança sobre a ameaça que representa o fato de possuir e especialmente de usar armas químicas contra a população, como uma reação enérgica por parte do governo da Síria, violando as normas e princípios mais 
elementares do direito internacional e do direito internacional humanitário. É preciso reconhecer que esta vez conseguiu-se um consenso unanime entre os membros do Conselho de Segurança para impor um processo de destruição das armas químicas, materiais e aditamentos que possui o Governo da República da Síria, mediante a Resolução do Conselho de Segurança 2118, sem ter que recorrer às sanções mencionadas no capítulo VII da Carta das Nações Unidas, pelo que imperou o direito internacional. Como poderá observar o leitor, as ações em conjunto tomadas tanto no seio do Conselho de Segurança, pela OPAQ e no consenso levado a cabo entre a Rússia e os Estados Unidos obrigaram ao governo da Síria a se comprometer a aceitar o estipulado na Convenção sobre Armas Químicas, e evitou-se desta forma que se realizara uma invasão militar à Síria, situação que começava a ser considerada pelo governo dos Estados Unidos.

Palavras-chave: Síria, armas químicas, Conselho de Segurança, Organização para a Proibição das Armas Químicas, Convenção sobre as Armas Químicas.

\section{Introducción}

El desafío en contra del régimen de Bashar al-Assad tuvo sus inicios en marzo de 2011, como consecuencia de lo que se conoció como la 'Primavera Árabe', ${ }^{1}$ movimiento social que tuvo como efecto directo el derrocamiento de gobiernos de países tales como Egipto y Túnez, mientras que, en Libia y en Siria, los gobiernos respondieron bélicamente. ${ }^{2}$ Entre los reclamos que ha hecho la población, hay que hacer énfasis en la exigencia para la creación de

\footnotetext{
1 Se le conoce como 'Primavera Árabe' a un conjunto de levantamientos y revoluciones que se dieron durante el período comprendido entre diciembre de 2010 y junio de 2011 en los países del norte de África y del Medio Oriente. La 'Primavera Árabe' en sí fue un movimiento con objetivos transformadores, tales como el derrocamiento de gobiernos y la implementación de una democracia, esto se dio en función de la dinámica interna de cada Estado en donde se presentaron acciones relacionadas con el movimiento. Por poner de ejemplos algunas acciones, podemos citar que, en Túnez, se dio la caída de Zayn al-Abidin Ben Ali, quién huyó a Arabia Saudita por la rebelión del pueblo tunecino; en Egipto, se dio la dimisión de Hosni Mubarak; mientras que, en Libia y en Siria, la situación fue diferente, ya que el gobierno de ambos Estados respondió militarmente en contra de las rebeliones que se dieron en sus respectivos territorios.

2 Ertuğrul, Doğan, “A test for Turkey's foreign policy: the Syria crisis”, Tesev's Foreign Policy Bulletin, 2012, The Turkish Economic and Social Studies Foundation, Estambul, pp. 1-2.
} 
un sistema político fundamentado en preceptos democráticos que le permita a la población saber que sus derechos fundamentales están protegidos; es decir que la crisis que se ha vivido en los últimos años en Siria se debe a varios factores cruciales, para que se pueda dar una reivindicación política y económica.

Como respuesta a las acciones del presidente Al-Assad, gobiernos como el de los Estados Unidos, Francia y Turquía han solicitado su renuncia ${ }^{3}$ y lo han puesto a discusión en el Consejo de Seguridad de las Naciones Unidas, reconociendo al mismo tiempo las Fuerzas de Oposición y la Coalición Nacional de la Revolución como el representante legítimo del pueblo de Siria. Hay que resaltar que una de las características del gobierno de Al-Assad ha sido el autoritarismo en contra de su población, $y$, como respuesta a sus demandas, el gobierno sirio ha usado la fuerza en contra de sus ciudadanos, reprimiendo militarmente aquellas protestas en contra del gobierno de AlAssad; este uso de la fuerza se ha caracterizado por ser excesivo, hasta el punto de usar armas químicas en contra de la propia población de Siria. ${ }^{4}$

\section{El uso de armas químicas en Siria}

Esta situación deja en entredicho la importancia de llevar a cabo una intervención militar en Siria; incluso, el gobierno de Al-Assad ha aceptado tener armas químicas, ${ }^{5}$ lo que se puede constatar en el Reporte de la Misión de las Naciones Unidas para Investigar las Denuncias de Empleo de Armas Químicas en la República Árabe Siria. ${ }^{6}$ En dicho reporte, se concluye el uso de este tipo de armas en un ataque perpetrado el 21 de agosto de 2013 en contra de los rebeldes en las áreas suburbanas de Damasco; sin embargo, el gobierno de Al-Assad negó categóricamente el uso de este tipo de armas. ${ }^{7}$ Desde esta

\footnotetext{
3 Sasnal, Patrycja, "The onset of civil war in Syria”, Bulletin, 2012, 339, (6), Polish Institute of International Affairs, Varsovia, p. 2.

4 "United Nations report on the alleged use of chemical weapons in the Ghouta area of Damascus on 21 August 2013", en http://www.un.org/disarmament/content/slideshow/ Secretary_General_Report_of_CW_Investigation.pdf

5 Comunicado: "La Casa Blanca se refiere a evaluación del uso de armas químicas por Siria", 30 de agosto de 2013, en http://iipdigital.usembassy.gov/st/spanish/texttrans/2013/08/20130830282038.html\#axzz2gfvhHzlO, consulta de octubre 3 de 2013.

6 http://www.un.org/disarmament/content/slideshow/Secretary_General_Report_of_ CW_Investigation.pdf

7 Nikitin, Mary Beth (coord.), Syria's chemical weapons: issues for Congress, Congressional Research Service, Working Paper R-43201, 2013.
} 
fecha, el Consejo de Seguridad ha insistido en investigar el uso de este tipo de armas para corroborar la versión oficial del gobierno de Siria, ya que, como es bien sabido, el uso de este tipo de armas viola, por un lado, el cumplimiento de la Convención en contra del Uso de Armas Químicas.

Conforme a los hechos que ocurrieron el 21 de agosto de 2013, se presentaron informes de inteligencia que confirmaron el uso de armas químicas por parte del régimen de Bashar Al-Assad; en dicho ataque, se contabilizaron más de 1.400 muertos, incluyendo una gran cantidad de niños, por lo que se calificó este acto como 'crimen contra la humanidad', ${ }^{8}$ lo que llevó al ejecutivo de los Estados Unidos de América a considerar una intervención militar que sería una 'acción limitada', militar se usaría solamente para responder al uso de armas de destrucción masiva por parte del gobierno de Siria en el conflicto de ese país, así como para detener el uso de tales armas en función de proteger los intereses de la seguridad nacional de los Estados Unidos de América y proteger a los aliados de este Estado en contra del uso de dichas armas y disminuir la capacidad de Siria para su utilización en un futuro. ${ }^{10}$ Situación que llevó a presentar ante el Congreso de los Estados Unidos de América una iniciativa donde se establece la posibilidad de realizar una intervención humanitaria en contra de Siria, con el objetivo de establecer control sobre las armas químicas que tiene bajo su poder el Estado sirio. ${ }^{11}$

\section{El uso de armas químicas: una amenaza para la paz y seguridad internacionales}

Como podemos ver, el uso de armas químicas y su proliferación constituyen una amenaza a la paz y seguridad internacionales, ${ }^{12}$ así como también una contravención a la Declaración de la Presidencia del Consejo de Seguridad realizada en enero de 1992, donde se hace énfasis de la obligación del control de armamento de destrucción masiva al afirmar que "los miembros

\footnotetext{
8 "Declaración del Secretario de Estado de los Estados Unidos sobre la situación de Siria", 30 de agosto de 2013, en http://iipdigital.usembassy.gov/st/spanish/texttrans/2013/08/20130830282055.html\#axzz2gfvhHzlO

$9 \quad$ Nikitin, Syria's chemical..., op. cit., p. 24.

10 S.J. Res. 21, Authorization for the use of military force against the government of Syria to respond to use of chemical weapons.

11 Ibíd.

12 UNSC, Resolución 1540 (2004).
} 
del Consejo destacan la necesidad de que todos los Estados miembros cumplan con sus obligaciones en relación con el control de armamentos y el desarme; eviten la proliferación en todos sus aspectos de todas las armas de destrucción en masa; eviten acumulaciones y transferencias excesivas y desestabilizadoras de armas...". ${ }^{13}$

Cabe mencionar que una declaración de la Presidencia del Consejo de Seguridad no equivale a una resolución y, por lo tanto, carece de carácter vinculante ${ }^{14}$ sin embargo, en ese tipo de declaración, se emite algún punto de vista, al cual los miembros del Consejo de Seguridad han llegado a través de un consenso. Así mismo, estas declaraciones sirven para invitar a los Estados a que tomen en cuenta y cumplan con lineamientos emitidos por el Consejo.

Igualmente, se violan los preceptos jurídicos establecidos en el Protocolo de Ginebra de $1925,{ }^{15}$ en donde se expresa lo siguiente: "Las altas partes contratantes, en tanto que no son ya partes en tratados que prohíben este empleo, reconocen esta prohibición, aceptan extender esta prohibición de empleo a los medios de guerra bacteriológicos y convienen en considerarse obligadas entre ellas en los términos de esta declaración". ${ }^{16}$

Para corroborar las graves acusaciones que se hicieron en contra del gobierno de Al-Assad, por el hecho de haber utilizado armas químicas en contra de la población siria, se decidió, por parte del Secretario General de las Naciones Unidas, crear una misión de investigación de acuerdo con lo establecido en la Resolución 42/37 de la Asamblea General, en donde se pide:

\begin{abstract}
Al Secretario General que, cuando un Estado miembro le proporcione información acerca de la posible utilización de armas químicas y bacteriológicas (biológicas) o toxínicas, que puedan constituir una transgresión del Protocolo de Ginebra de 1925 o de otras normas pertinentes al derecho internacional consuetudinario, lleve a cabo investigaciones a fin de completar las averiguaciones del caso e informe prontamente a todos los Estados miembros de los resultados de esas investigaciones.
\end{abstract}

\footnotetext{
13 UnSC, Nota del Presidente del Consejo de Seguridad, Doc. S/23500 (21 de enero de 1992).

14 UNSC, Reglamento, artículo 39.

15 La República Árabe de Siria se adhirió a este protocolo en 1968.

16 Protocolo de Ginebra sobre Prohibición del Empleo en la Guerra de Gases Asfixiantes, Tóxicos o Similares o Procedimientos Análogos. (17 de junio de 1925).
} 
Del mismo modo, como en ocasiones anteriores, el Consejo de Seguridad hace la sugerencia al Secretario General de las Naciones Unidas para que lleve a cabo investigaciones como una respuesta a las denuncias que se reciban en atención de la posibilidad de que hayan sido usadas armas químicas y/o biológicas. ${ }^{17}$ La creación de la misión obedece a la necesidad de determinar si realmente las denuncias sobre el uso de armas químicas están fundamentadas en datos verídicos.

\section{La cooperación entre la ONU y la OPAQ}

El Secretario General de las Naciones Unidas solicitó la intervención de la Organización para la Prohibición de las Armas Químicas (OPAQ) en una reunión que se llevó a cabo el 28 de agosto de 2013, apenas siete días después del ataque en Damasco, teniendo como cometido crear una misión en la cual, de manera imparcial y objetiva, pudiera establecer los hechos sobre el uso de armas químicas en Siria. ${ }^{18}$ Considerando de esta manera la cooperación "en cualquier cuestión que se refiera al objetivo y el propósito de la Convención (la Convención sobre la Prohibición del Desarrollo, la Producción, el Almacenamiento y el Empleo de Armas Químicas y sobre su Destrucción) o se plantee en relación con su cumplimiento", ${ }^{19}$ a la cual Siria se adhirió el 14 de septiembre de 2013.

Así mismo, cabe mencionar que la cooperación entre la OPAQ y la Organización de las Naciones Unidas (ONU) en el caso de Siria tiene su fundamento legal conforme al reconocimiento en función de las actividades que se enfocan a la realización de actividades, cuyo objetivo es lograr la prohibición total de las armas químicas, ${ }^{20}$ según lo que dicta la Convención sobre la Prohibición del Desarrollo, la Producción, el Almacenamiento y el Empleo de Armas Químicas y sobre su Destrucción (CAQ). ${ }^{21}$

\footnotetext{
17 UNSC, Resolución 620 (1988).

18 “UN Secretary-General, OPCW Director-General Meet in The Hague", en http://www. opcw.org/sp/news/article/un-secretary-general-opcw-director-general-meet-in-the-hague/ 19 UNGA, Resolución 55/283, Anexo, Acuerdo de Relación entre las Naciones Unidas y la Organización para la Probibición de las Armas Químicas, artículo II, fracción 2, inciso f (24 de septiembre de 2001).

20 Ibíd.

21 La Convención sobre la Prohibición del Desarrollo, la Producción, el Almacenamiento y el Empleo de Armas Químicas y sobre su Destrucción entró en vigor en 1997.
} 
La cooperación entre la ONU y la OPAQ se enfocará especialmente en aquellos casos en donde exista presunción de gravedad y urgencia, por lo que la OPAQ puede examinar dudas sobre el cumplimiento de la Convención y está facultada para solicitar al Estado medidas para subsanar la situación en donde sea patente la falta de cumplimiento de la CAQ. Entre las medidas que la OPAQ pudiera tomar, se encuentra la formulación de recomendaciones a la Conferencia ${ }^{22}$ respecto a las disposiciones que pudieran subsanar la situación y, sobre todo, asegurar el cumplimiento de estas. ${ }^{23}$

\section{La misión de investigación sobre el uso de armas químicas}

La misión llegó desde el 18 de agosto a Siria y, a pesar de que su mandato indicaba que se hicieran inspecciones en Khan al-Asal, Sheik y Maqsood Saraqeb, resulta que estaban presentes en dicho país en el momento en que se realizaron los ataques del 21 de agosto de 2013. Ante esta circunstancia, el Secretario General de las Naciones Unidas le solicitó a la misión que las investigaciones sobre el uso de armas químicas fueran enfocadas en la zona de Ghouta, en Damasco, por lo que se procedió a efectuar inspecciones in situ en dicha zona de la capital siria.

Al momento de hacer la investigación in situ, por parte de la misión, se localizaron cohetes capaces de transportar cargas químicas y se comprobó que la mayoría de fragmentos de cohetes contenían sarín, ${ }^{24}$ además de que algunos de los sobrevinientes mostraban síntomas que correspondían a una intoxicación con fosfatos orgánicos $;{ }^{25}$ igualmente, se tomaron muestras de sangre, cabello y orina de los sobrevivientes, las cuales dieron positivo a las

22 Convención sobre la Prohibición del Desarrollo, la Producción, el Almacenamiento y el Empleo de Armas Químicas y sobre su Destrucción, artículo VIII, fracción 9 (13 de enero de 1993).

23 Ibíd., artículo VIII, fracción 36.

24 El sarín, conocido también como GB, es un agente bélico químico artificial clasificado como agente nervioso. Los agentes nerviosos son los más tóxicos y de más rápida acción entre los agentes bélicos químicos conocidos. En http:/ /www.ndhan.gov/data/translation/ Sarin $\% 20$ Nerve $\% 20$ Gas-Spanish.pdf

25 El gas sarín es similar a ciertas clases de pesticidas denominados organofosfatos, en términos de cómo funcionan y de qué tipo de efectos perjudiciales causan. No obstante, los agentes nerviosos son mucho más potentes que los pesticidas organofosfatos. En http:// www.ndhan.gov/data/translation/Sarin\%20Nerve\%20Gas-Spanish.pdf 
señales de intoxicación, proporcionando pruebas positivas de la exposición al sarín de los sobrevivientes que fueron estudiados por la misión. ${ }^{26}$ En este sentido, la misión concluyó que, durante el ataque del 21 de agosto de 2013, en Ghouta, efectivamente se hizo uso de armas químicas, las cuales afectaron a civiles, incluyendo niños. Las muestras químicas, ambientales y médicas que la misión había recogido y analizado proporcionan pruebas contundentes y convincentes de que en dicha zona se utilizaron cohetes tierra a tierra que contenían el agente neurotóxico denominado sarín. ${ }^{27}$

\section{La posibilidad de hacer uso de la fuerza por parte de los Estados Unidos}

Cabe mencionar que incluso antes de que se tuviera la información puntual de la misión, los Estados Unidos ya se estaban preparando para hacer uso de la fuerza militar en el territorio de Siria; y, conforme a este criterio, el presidente Barack Obama envió al Congreso de los Estados Unidos de América un proyecto de resolución en donde se hacía énfasis en los argumentos sobre los cuales se basa la posibilidad de hacer el uso de la fuerza. Por una parte, se argumenta que 1) se debe prevenir o detener el uso o la proliferación (incluyendo la transferencia a grupos terroristas $u$ otros actores estatales o no estatales) dentro, o proveniente de Siria, de cualquier arma de destrucción masiva, incluyendo armas químicas o biológicas o sus componentes o materiales usados en dichas armas; 2) proteger a los Estados Unidos de América o a sus aliados contra la amenaza planteada por dichas armas. ${ }^{28}$

Así mismo, un evento similar se dio en el Senado de los Estados Unidos: el Comité de Relaciones Exteriores debatió y adoptó la Resolución $21,{ }^{29}$ en donde se autorizaba al Presidente de los Estados Unidos a hacer uso de la fuerza bajo las siguientes circunstancias: 1) responder al uso de armas de destrucción masiva por parte del gobierno de Siria en el conflicto en ese país; 2) detener el uso de tales armas por parte de Siria en orden de proteger

\footnotetext{
26 UN, Secretaría General, Informe de la misión de las Naciones Unidas encargada de investigar las denuncias relativas al empleo de armas químicas en la República Árabe Siria acerca del incidente ocurrido en la zona de Ghouta, en Damasco, el 21 de agosto de 2013, Doc. A/67/997, S/2013/553 (16 de septiembre de 2013).

27 Ibíd.

28 Miller, Vaughne, US Government and Congress Response to Syria 8 (House of Commons, Standard Note: SNIA 6721), 2013.

29 S.J., Res. 21, Authorization for the use of military force against the government of Syria to respond to use of chemical weapons.
} 
la seguridad nacional de los Estados Unidos y proteger a los aliados de los Estados Unidos contra el uso de dichas armas; 3) disminuir la capacidad de Siria del uso de dichas armas en el futuro; y 4) prevenir la transferencia a grupos terroristas o a otro Estado o actores no estatales dentro de Siria de cualquier tipo de arma de destrucción masiva. ${ }^{30}$

A pesar de que el Comité de Relaciones del Senado fundamenta el uso de la fuerza militar bajo preceptos tales como que "1) los Estados Unidos han usado todos los medios diplomáticos apropiados y otros medios pacíficos para prevenir el desarrollo; 2) el gobierno ha llevado a cabo uno o más ataques significativos con armas químicas; 3) el uso de la fuerza es necesario para responder el uso de armas químicas por parte del gobierno de Siria; 4) está en la esencia de los intereses de seguridad nacional de los Estados Unidos el uso de la fuerza militar", ${ }^{31}$ sin embargo, cabe mencionar que estos preceptos no tuvieron suficiente peso ante la comunidad internacional para apoyar algún tipo de intervención militar por parte de los Estados Unidos de América.

\section{6. ¿Derecho norteamericano versus derecho internacional?}

Como se puede observar, los Estados Unidos estaban tomando todas las consideraciones necesarias para realizar una intervención militar, aunque limitada, pero no deja de ser una intervención militar; y esta toma de decisiones se realizaba bajo un esquema unilateral conforme a su derecho interno, ${ }^{32}$ disminuyéndole importancia a la doctrina y a la jurisprudencia internacional, así como al derecho internacional en su conjunto.

Lo más preocupante de esta situación son las posibles acciones que pudieran tomar los Estados Unidos de América, ya que algunas aseveraciones hechas por el presidente Obama son violatorias al derecho internacional. Por tomar algunos ejemplos, en la declaración del Presidente realizada el 31 de agosto de 2013, hace énfasis sobre la decisión de que "los Estados Unidos deberían tomar una acción militar en contra de objetivos del gobierno

\footnotetext{
30 Ibíd., p. 4.

31 Ibíd., p. 5.

32 Ver los siguientes documentos: Resolución 1541, Resolución 1547 y Resolución 1544 del Congreso de los Estados Unidos.
} 
de Siria". ${ }^{33}$ Si lo vemos sobre esta perspectiva simplista, podemos observar que se trata de una afirmación sobre una intervención militar unilateral, en ningún momento se afirma la posibilidad de legitimar esta acción a través de obtener un consenso por parte de la Comunidad Internacional, sino, al contrario, el Presidente afirmó: "No espero que todas las naciones estén de acuerdo con la decisión que nosotros tomemos". ${ }^{34}$

Con las afirmaciones anteriores, vemos que, si se llevan a cabo las acciones consideradas, se estaría violando el artículo $2^{\circ}$, párrafo 4 , de la Carta de las Naciones Unidas, que menciona lo siguiente: "Los miembros de la Organización, en sus relaciones internacionales, se abstendrán de recurrir a la amenaza o al uso de la fuerza contra la integridad territorial o la independencia política de cualquier Estado, o en cualquier otra forma incompatible con los propósitos de las Naciones Unidas".

Así como también el principio de derecho internacional de la proscripción del uso de la fuerza: "a) El principio de que los Estados, en sus relaciones internacionales, se abstendrán de recurrir a la amenaza o al uso de la fuerza contra la integridad territorial o la independencia política de cualquier Estado, o en cualquier otra forma incompatible con los propósitos de las Naciones Unidas". ${ }^{35}$

El uso de la fuerza constituye per se una flagrante violación del derecho internacional y ha quedado estipulado, tanto en la Carta de las Naciones Unidas como en la Declaración sobre los Principios de Derecho Internacional Referentes a las Relaciones de Amistad y a la Cooperación entre los Estados de Conformidad con la Carta de las Naciones Unidas, que no se debe emplear para resolver cuestiones internacionales, incluyendo aquellos actos de represalia que impliquen el uso de la fuerza. ${ }^{36}$ Ahora bien, en la declaración antes citada, queda asentado que "ningún Estado o grupo de Estados tiene derecho a intervenir directa o indirectamente, y sea cual fuere el motivo". ${ }^{37}$

\footnotetext{
33 "Declaración del Presidente de los Estados Unidos sobre Siria", 31 de agosto de 2013, en http://www.whitehouse.gov/the-press-office/2013/08/31/statement-president-syria, consulta de noviembre 6 de 2013.

34 Ibíd.

35 UNGA, Res. 2625 (XXV), Declaración sobre los Principios de Derecho Internacional Referentes a las Relaciones de Amistady a la Cooperación entre los Estados de Conformidad con la Carta de las Naciones Unidas, Resolución de la Asamblea General (24 de octubre 24 de 1970).

36 Ibíd.

37 Ibíd.
} 
En el momento en que el presidente Obama emite una declaración sobre la situación en Siria da entender que está dispuesto a llevar a cabo una intervención militar sin el consentimiento del Consejo de Seguridad al mencionar lo siguiente: "Me siento a gusto yendo hacia adelante, sin la aprobación del Consejo de Seguridad de las Naciones Unidas", ${ }^{38}$ haciendo referencia a la posibilidad de poner en práctica el plan de realizar una intervención militar limitada, como lo menciona la resolución aprobada por el Senado 'S.J., Res. 21, Authorization for the use of military force against the government of Syria to respond to use of chemical Weapons', lo que sería una grave violación a la Carta de las Naciones Unidas, ya que desconocería los poderes de este órgano, conforme lo estipulado en la misma Carta: "A fin de asegurar acción rápida y eficaz por parte de las Naciones Unidas, sus miembros confieren al Consejo de Seguridad la responsabilidad primordial de mantener la paz y la seguridad internacionales, y reconocen que el Consejo de Seguridad actúa a nombre de ellos al desempeñar las funciones que le impone aquella responsabilidad". ${ }^{39}$

A pesar de la postura del presidente Obama, hay analistas políticos de los Estados Unidos que apoyan la necesidad de llegar a una solución política conforme al conflicto, asegurando las armas químicas, sin que exista la necesidad de desencadenar un conflicto armado; ${ }^{40}$ ya que el simple hecho de considerar realizar una intervención armada puede traer problemas más graves, como represalias a este tipo de acciones, por ejemplo, ataques terroristas o atrocidades a la población de Siria.

Por otra parte, también se hacen críticas a la posible inacción, lo cual puede contribuir al crecimiento de la crisis humanitaria en Siria, aparte de argumentar que la credibilidad global de los Estados Unidos está siendo menguada por la reticencia por parte del gobierno de Al-Assad en cuanto a terminar los violaciones de los derechos fundamentales de los ciudadanos sirios, a pesar de las demandas de la comunidad internacional.

La inacción por parte de la comunidad internacional ante el conflicto en Siria se vería como una contravención a los preceptos abordados sobre la responsabilidad de proteger, dado que, como se menciona en el párrafo anterior, si la comunidad internacional no reacciona frente a la crisis humanitaria, se corre el riesgo de que esa crisis se convierta en una situación más

\footnotetext{
38 "Declaración del Presidente de los Estados Unidos sobre Siria", op. cit.

39 Carta de las Naciones Unidas, artículo 24, párrafo 1.

40 Nikitin, Syria's chemical..., op. cit., p. 5.
} 
grave. Para esto, hay que hacer mención de lo que significa la responsabilidad de proteger.

La idea de realizar un estudio sobre el dilema que se enfrentaba, la soberanía de los Estados versus las intervenciones humanitarias, nació después de que el entonces secretario de la ONU, el Sr. Kofi Annan, instó a los Estados a que se hicieran valer los principios de la Carta y que se defendieran aquellos criterios de la humanidad que sean comunes para todos los Estados. Por ello, se realizó el siguiente cuestionamiento: “... si la intervención humanitaria es, en realidad, un ataque inaceptable a la soberanía, ¿cómo deberíamos responder a situaciones como las de Rwanda y Srebrenica, y a las violaciones graves y sistemáticas de los derechos humanos que transgreden todos los principios de nuestra humanidad común?”. ${ }^{41}$

En respuesta al cuestionamiento realizado por el entonces Secretario General de la ONU, el gobierno de Canadá tomó la iniciativa para crear la Comisión Internacional sobre Intervención y Soberanía de los Estados (CIISE). El objetivo principal de esta Comisión era llegar a un punto de acuerdo entre los fines de la protección humanitaria y la soberanía de los Estados.

Los conflictos armados, como el que se presentó en Siria, presenta un fuerte dilema de la comunidad internacional: por un lado, está la situación en donde los Estados intervengan en el conflicto con la intención de frenar los abusos; sin embargo, se corre el riesgo de que el o los Estado(s) que intervengan puedan llegar a tomar partido en el conflicto interestatal, aumentando la gravedad de este conflicto. Y, por otro lado, se puede caer en la inacción y mantenerse al margen de este conflicto, convirtiéndose, por omisión, en un cómplice indirecto de las masacres que se estén suscitando en el conflicto.

Lo más delicado de autorizar una intervención con fines humanitarios no se trata de quién la autoriza, ni cuando es autorizada la intervención, sino cómo debe ser autorizada la intervención humanitaria y tener los objetivos bien claros.

En el caso de Siria, se optó por no realizar una intervención, sino someter al gobierno Sirio a acatar una serie de obligaciones hacia su población para evitar o al menos disminuir el derramamiento de sangre en ese Estado.

41 UNGA, Informe del Secretario General A/54/2000, titulado: Nosotros los pueblos: la función de las Naciones Unidas en el siglo XXI. 


\section{Objetivo de la comunidad internacional: destruir las armas químicas evitando cualquier intervención armada}

Con el objetivo de evitar cualquier tipo de intervención, incluyendo la intervención armada, ya fuera en ese momento o en un futuro próximo por parte de los Estados Unidos o cualquier Estado que tuviera el interés de realizar una intervención, la Liga de Estados Árabes envió una misiva al Secretario General de las Naciones Unidas fechada el día 22 de enero de 2012, en donde se mencionaba que "los ciudadanos pensaban que la crisis se debía resolver de manera pacífica, únicamente con mediación árabe, sin intervención internacional. Ello les permitiría vivir en paz y concluir el proceso de reforma y lograr los cambios que desean". ${ }^{42}$

Esta misma postura se fue reforzando en el seno del Consejo de Seguridad, como se puede ver en la posición que tomó el Estado de Qatar al afirmar: "No estamos pidiendo una intervención militar; estamos abogando por la adopción de una presión económica concreta a fin de que el régimen sirio entienda que no puede hacer caso omiso de las demandas de su pueblo". ${ }^{43}$

Del mismo modo, la Liga de Estados Árabes, a través de su Secretario General, daba a conocer la postura que se tenía con respecto a Siria, en donde se abordaba la problemática de Siria desde un punto de vista regional, con base en un contexto árabe; por consiguiente, se le solicitaba al gobierno de Al-Assad las demandas del pueblo sirio en cuestión de cambio económico, social y político, así como el respeto de los derechos fundamentales de la población, pero, sobre todo, se buscaba evitar cualquier intervención extranjera, específicamente la intervención militar. ${ }^{44}$

La posible intervención de los Estados Unidos a Siria se enfoca en la contribución de que estos pudieran controlar el desarme de armas químicas, sin embargo, tanto Rusia como China se oponían a la iniciativa de los Estados Unidos a usar cualquier medio militar para contrarrestar los hechos ocurridos en Siria, por lo que el gobierno de Rusia presentó una propuesta relacionada con el desarme de las armas químicas en Siria.

Incluso Rusia, antes que se hubiera obtenido el informe de la misión y a pesar de que este Estado se oponía a la intervención militar en Siria, dejó

\footnotetext{
42 UNSC, Carta de fecha 24 de enero de 2012 dirigida al Presidente del Consejo de Seguridad por el Secretario General, Doc. S/2012/71 (30 de enero de 2012).

43 UnSC, Sesión $6710^{a}$, Doc. S/PV.6710 (31 de enero de 2012).

44 Ibíd.
} 
abierta la posibilidad de participar en una operación militar, siempre y cuando se demuestre que se usaron armas químicas y con la aprobación del Consejo de Seguridad. ${ }^{45}$ Desde esta misma óptica, el representante en conjunto de la ONU y de la Liga Árabe de Estados para Siria, el Sr. Lakhdar Brahimi, avaló la aseveración realizada por Rusia, al mencionar que "cualquier acción militar con ese país [Siria] debe ser autorizada por el Consejo de Seguridad”. "China también mostró una postura en contra de un ataque militar, por lo que Xi Jinping, presidente de este país, aseveró: “Una solución política es el único camino correcto para la crisis en Siria y un ataque militar no puede resolver el problema desde la raíz". ${ }^{47}$ Estas declaraciones, tanto la del Representante en Conjunto, la del gobierno de Rusia y la del Presidente de China, se dieron antes de tener los informes de la misión. ${ }^{48}$

\section{La colaboración entre los Estados Unidos y Rusia}

Días previos a que se emitieran los informes por parte de la misión, los Estados Unidos y Rusia expresaron su determinación en conjunto para asegurarse de la destrucción de las armas químicas de Siria, de la manera más rápida y segura, y buscar obtener una declaración por parte de la OPAQ, por lo que estas dos potencias expresaron su compromiso de trabajar en conjunto para obtener una resolución en el seno del Consejo de Seguridad, que reforzara las acciones de la OPAQ con respecto al procedimiento que se acordaría para destruir las armas químicas en Siria, ${ }^{49}$ formulando un draft para la eliminación de las armas químicas en ese país.

En ese esbozo, se menciona que la resolución obtenida del debate en el Consejo de Seguridad debería tener los procesos para asegurarse la verificación y la correcta implementación, y que se le solicitaría al Secretario

\footnotetext{
45 Radio La Voz Rusa, 4 de septiembre de 2013, en http://spanish.ruvr.ru/ news/2013_09_04/Putin-admite-la-posibilidad-de-acuerdo-de-Rusia-con-una-operaci-nmilitar-en-Siria-9071/

46 Centro de Noticias de la ONU, "Siria: Brahimi advierte que cualquier acción militar debe ser autorizada por la ONU”, en http://www.un.org/spanish/News/story.asp?NewsID=27309\#. U11xwRAuekc

47 Agencia Reuters, “China's Xi tells Obama Syria crisis can't be resolved with military strike", en http://www.reuters.com/article/2013/09/06/us-syria-crisis-china-idUSBRE9850BY20130906

48 La misión presentó sus informes el 16 de septiembre de 2013.

49 OPAQ, Doc. EC-M-33/NAT.1, Anexo.
} 
General de las Naciones Unidas, en conjunto con la OPAQ, que enviara las recomendaciones pertinentes al Consejo de Seguridad sobre la eliminación de las armas químicas en Siria, dejando abierta la posibilidad de imponer medidas conforme a lo establecido en el capítulo VII de la Carta de las Naciones Unidas. ${ }^{50}$

El fundamento jurídico en el que se basaba el esbozo de Rusia y de los Estados Unidos era obviamente la Convención sobre las Armas Químicas de 1997, en cumplimiento con el acto jurídico internacional de la adhesión de Siria a esta Convención, acto que se llevó a cabo el 14 de septiembre de 2013. ${ }^{51}$ Específicamente, se hace alusión a lo contenido en el artículo IV, párrafo 8, que enuncia lo siguiente: "8. Si un Estado ratifica la presente Convención o se adhiere a ella después de transcurrido el período de diez años establecido para la destrucción en el párrafo 6, destruirá las armas químicas especificadas en el párrafo 1 lo antes posible. El Consejo Ejecutivo determinará el orden de destrucción y el procedimiento de verificación estricta para ese Estado parte".

Así mismo, se tomó en cuenta el artículo V, párrafo 10, que habla sobre la destrucción de las instalaciones: "10. Si un Estado ratifica la presente Convención o se adhiere a ella después de transcurrido el período de diez años establecido para la destrucción en el párrafo 8, destruirá las instalaciones de producción de armas químicas especificadas en el párrafo 1 lo antes posible. El Consejo Ejecutivo determinará el orden de destrucción y el procedimiento de verificación estricta para ese Estado parte".

Por lo tanto, también se hace énfasis en la total cooperación de Siria ante la decisión que se tome por parte de la OPAQ en cuanto a su implementación se refiere; igualmente, se menciona que se debe establecer un programa para la destrucción expedita de las armas químicas, considerando las siguientes fechas:

a) La terminación de las inspecciones de los sitios declarados in situ por la OPAQ, en noviembre.

b) Destrucción del equipo producción, mezclado/llenado, en noviembre.

\footnotetext{
50 Ibíd.

51 "Estados miembros de la Organización para la Prohibición de las Armas Químicas (OPAQ)", en http://www.opcw.org/nc/sp/acerca-de-la-opaq/estados-miembros/?tx_opcwmemberstate_pi1[sortField] $=0 \&$ tx_opcwmemberstate_pi1 [sortReverse] $=0 \&$ tx_opcwmemberstate_pi1[ASP_PAGE] $=2 \&$ tx_opcwmemberstate_pi1 $[$ pointer $]=0$
} 
c) Eliminación completa de todo el material de las armas químicas y su equipamiento, en la primera mitad del año $2014 .^{52}$

De la misma forma, en el draft se hacía énfasis en que la decisión que se tome por la OPAQ debe considerar algunas cuestiones, tales como la verificación de las medidas impuestas, la rapidez con la que se deban empezar las acciones por parte de la OPAQ, así como también, en caso necesario, todos aquellos requerimientos especiales y suplementarios, al igual que el apoyo de todos los Estados que pudieran colaborar de una manera relevante para el fin específico expuesto en la decisión.

\section{La decisión de la OPAQ}

Considerando el trabajo previo realizado en conjunto por Rusia y los Estados Unidos, se obtuvo la Decisión de la Destrucción de las Armas Químicas en Siria por parte de la OPAQ, ${ }^{53}$ en donde se decide de manera institucional el procedimiento por seguir para la destrucción de las armas antes mencionadas; de igual manera, se resalta que "el uso de armas químicas por alguien, bajo cualquier circunstancia, podría ser reprensible y completamente contrario a las normas legales y estándares de la comunidad internacional". ${ }^{54}$

En la decisión, se hace 'la invitación' al gobierno de Siria para que reciba a la delegación de la OPAQ, así como también para que coopere con la OPAQ en aquello que se estime necesario para la aplicación de la Convención, pero teniendo en cuenta que el Consejo Ejecutivo someterá el caso y toda aquella información pertinente a la Asamblea General y al Consejo de Seguridad de las Naciones Unidas, ${ }^{55}$ corroborando la colaboración entre la OPAQ y la ONU mediante el Acuerdo de Cooperación entre las Naciones Unidas y la Organización para la Prohibición de las Armas Químicas. ${ }^{56}$

Por lo tanto, conforme a la decisión emitida por el Consejo Ejecutivo de la OPAQ, se determinó la orden de destrucción y de procedimientos para

\footnotetext{
52 OPAQ, Doc. EC-M-33/NAT.1, Anexo A.

53 OPAQ, Doc. EC-M-33/DEC.1.

54 Ibíd.

55 Convención sobre la Prohibición del Desarrollo, la Producción, el Almacenamiento y el Empleo de Armas Químicas y sobre su Destrucción, artículo VIII, fracción 36 (13 de enero de 1993).

56 UNGA, Resolución 55/283, Anexo, Acuerdo de Relación entre las Naciones Unidas y la Organización para la Probibición de las Armas Químicas (24 de septiembre de 2001).
} 
su estricta verificación, en donde destacaban aspectos como la entrega de la lista de nombres de los químicos, toxinas y cantidades que se tienen; el tipo de municiones específicas, submuniciones, dispositivos que integran las armas químicas; la locación de todas las armas químicas, los almacenes de las armas químicas, las instalaciones de las plantas productoras de las armas químicas; las instalaciones donde se realizan las investigaciones y desarrollo de las armas químicas, proveyendo las coordenadas geográficas específicas, ${ }^{57}$ con el objetivo de destruir todas las armas químicas, todo el material que se utiliza en su fabricación y todo el equipamiento, en la primera mitad del año $2014,{ }^{58}$ tal y como se sugirió en el esbozo presentado por Rusia y los Estados Unidos.

\section{La Resolución 2118 del Consejo de Seguridad}

En la 7038 a Sesión del Consejo de Seguridad, se optó por emitir la Resolución 2118, que, en realidad, se trata de una resolución jurídicamente vinculante, con la finalidad de que Siria cumpla con sus obligaciones jurídicas, tanto en el ámbito interno como en sus compromisos internacionales. Esta resolución es el primer paso para poder llegar a una solución en el conflicto de Siria, como lo expresó el secretario general de las Naciones Unidas, Ban Ki-Moon, al señalar: "Llevo muchos meses diciendo que, de confirmarse el uso de armas químicas en Siria, habría que dar una respuesta unida y firme. Esta noche, la comunidad internacional la ha dado [...] Puesto que la misión que fue enviada a investigar las denuncias confirmó el uso de armas químicas en Siria, los autores de este crimen deben ser llevados ante la justicia".

Uno de los aspectos más importantes que se buscaba obtener con la resolución es la eliminación lo más rápido posible de las armas químicas que Siria posee, con la mayor transparencia y bajo los lineamientos y vigilancia de la OPAQ y de las Naciones Unidas en conjunto y de manera cooperativa, así como también se espera el apoyo de la comunidad internacional. ${ }^{59}$ Otro de los objetivos que se pretende con la resolución es que tanto el gobierno de Siria como la oposición respeten las normas del derecho internacional humanitario. ${ }^{60}$

\footnotetext{
57 OPAQ, Doc. EC-M-33/DEC.1.

58 Ibíd.

59 Ibíd.

60 UNSC, Resolución 2118 (2013).
} 
La Resolución 2118 del Consejo de Seguridad se obtuvo gracias a la colaboración entre los Estados Unidos y Rusia, que, pese a que ambos gobiernos tenían ideas divergentes para solucionar el problema, finalmente llegaron a un acuerdo en las reuniones celebradas en Ginebra el 14 de septiembre de 2013; ${ }^{61}$ incluso, el mismo Secretario de Estado de los Estados Unidos mencionó que "el Consejo de Seguridad ha demostrado que la diplomacia puede ser tan potente como para neutralizar las peores armas de guerra", ${ }^{2}$ después de que el gobierno de los Estados Unidos había expresado su intención de realizar un ataque militar para la destrucción de las armas químicas. ${ }^{63}$ De todas formas, dejó en entredicho la posibilidad de un ataque militar como una manera de frenar la capacidad de Siria con respecto a las armas químicas, al aseverar que "la opción de la fuerza militar que el presidente Obama ha mantenido sobre la mesa hubiera podido dar resultado en ese sentido, pero, en realidad, con la resolución [...] se consigue incluso más". ${ }^{64}$ Aun así reconoce que por primera vez se tratará de eliminar la capacidad de una nación con respecto a las armas químicas por medios pacíficos. ${ }^{65}$

Uno de los aspectos más importantes que hay que resaltar es que, a pesar de haber emitido una resolución vinculante, no se acoge al capítulo VII de la Carta de las Naciones Unidas y, por lo tanto, no se menciona ningún tipo de acciones coercitivas para garantizar el cumplimiento de lo dispuesto en la resolución, por lo que la solución de la problemática de las armas químicas se intenta desde la vía política y diplomática, tal y como se había planteado en junio de 2012. ${ }^{66}$ Así mismo, se tiene la creencia de que el gobierno de Siria pueda tener disposición de colaborar activamente con los inspectores de la OPAQ y con las Naciones Unidas, esto se debe principalmente a la disposición a adherirse a la Convención sobre Armas Químicas.

Sin embargo, no se descarta totalmente la aplicación del capítulo VII de la Carta de las Naciones Unidas, en caso de que se lleguen a violar las

61 Blog oficial del Departamento de Estado, A framework for the elimination of Syrian chemical weapons, 15 de septiembre de 2013, en http://blogs.state.gov/stories/2013/09/15/ framework-elimination-syrian-chemical-weapons

62 unsC, Sesión 7038ª , Doc. S/PV. 7038 (27 de septiembre de 2013).

63 "Declaración del Presidente de los Estados Unidos sobre Siria", op. cit.

64 UNSC, Sesión 7038ª Doc. S/PV. 7038 (27 de septiembre de 2013).

65 Ibíd.

66 unsC, Documento del Consejo de Seguridad, Informe del Secretario General relativo a la aplicación de la Resolución 2043 (2012), Doc. S/2012/523 (6 de julio de 2012). 
condiciones expresas en las Resolución $2118,{ }^{67}$ ya que existe el temor de que aquellos grupos opositores al régimen de Al-Assad pudieran entregar este tipo de armas a grupos no estatales con tendencias extremistas. ${ }^{68}$

El hecho de obtener la Resolución 2118 reflejó la confianza hacia las organizaciones internacionales y hacia las normas internacionales, que en el discurso del presidente Obama del 31 de agosto de 2013, se vio un poco minada y dejó en duda la labor del Consejo de Seguridad, al igual que en la resolución obtenida por el Senado de los Estados Unidos, la cual no contempla las normas del derecho internacional para actuar conforme a los lineamientos militares. ${ }^{69}$

Entre los aspectos destacables de la Resolución 2118, se menciona que efectivamente el empleo de armas químicas por parte de Siria es una amenaza para la paz y seguridad internacionales; no obstante, no se hace alusión a las medidas mencionadas en el capítulo VII de la Carta de las Naciones Unidas (como ya se había mencionado anteriormente), sino que se hace énfasis sobre la solución de la crisis en Siria, la cual se basa en un proceso político inclusivo, ${ }^{70}$ por lo que prohíbe a Siria el empleo, desarrollo, producción o almacenaje de armas químicas, ya sea con la ayuda de otros Estados o actores no estatales, por lo que se compromete a Siria a colaborar activamente a proporcionar toda la ayuda necesaria para los miembros de la OPAQ y de las Naciones Unidas involucrados en la destrucción de las armas químicas, así como también debe realizar un informe mensual para el Consejo de Seguridad sobre el desarrollo de la aplicación de la resolución, especialmente los progresos que se vayan logrando con su cumplimiento, al igual que en lo dispuesto en la Convención sobre Armas Químicas.

\section{A modo de conclusión}

Como se ha podido observar, la emisión de una resolución por parte del Consejo de Seguridad logró un consenso para obligar al gobierno de Siria a que, en un período determinado de tiempo, se lleve a cabo un proceso de

\footnotetext{
67 UNSC, Sesión 7038 , Doc. S/PV. 7038 (27 de septiembre de 2013).

68 Zeit Online, “Syriens Giftgasbestände sollen größtenteils nicht waffenfähig sein”, 27 de septiembre de 2013, en http://www.zeit.de/politik/ausland/2013-09/syrien-giftgas-fluessigkeit, consulta de noviembre 6 de 2013.

69 S.J., Res. 21, Authorization for the use of military force against the government of Syria to respond to use of chemical weapons.

70 UNSC, Resolución 2118 (2013).
} 
destrucción de armas químicas, impidiendo el uso de este tipo de armas como medio coercitivo hacia su población; sin embargo, esto es solo un paso en la búsqueda por la solución al conflicto que se vive actualmente en Siria.

Cabe aclarar que, aunque no se haya hecho uso de las sanciones mencionadas en el capítulo VII de la Carta de las Naciones Unidas, no se puede descartar totalmente la idea de su uso, sobre todo en el caso de la crisis humanitaria, pero, al menos, no se llevó a cabo una intervención militar por parte de los Estados Unidos de América, a pesar de que se había tomado en consideración e, incluso, se había aprobado por el Senado de este Estado. Pues esta situación contravendría los principios del derecho internacional y la Carta de las Naciones Unidas al hacer uso de la fuerza de manera unilateral, sin una autorización expresa por parte del Consejo de Seguridad; es decir que, ante esta situación, predominó el consenso de los miembros del Consejo de Seguridad al evitar una intervención militar, al menos por el momento, y obligar al Estado sirio a que lleve a cabo una serie de medidas, como la adhesión a la Convención sobre la Prohibición del Desarrollo, la Producción, el Almacenamiento y el Empleo de Armas Químicas y sobre su Destrucción, y la orden de destrucción de las armas químicas, así como de los componentes y los lugares donde se ensamblaban.

A pesar de que se consumaron hechos lamentables por parte del gobierno de Siria al usar armas químicas en contra de su población, se logró utilizar los instrumentos jurídicos internacionales para evitar que se siguieran empleando este tipo de armas y, sobre todo, se consiguió que se destruyera casi todo el arsenal de armas químicas que Siria mantenía en su poder, según un informe presentado por la titular de la misión conjunta de la ONU y la OPAQ al Consejo de Seguridad, en septiembre de 2014.

La aplicación del derecho internacional en el conflicto que se presenta en Siria debe enfocarse a evitar que la crisis actual vaya en aumento; esperemos que el proceso político sugerido por la comunidad internacional para la solución del problema en Siria pueda ser claro, preciso y objetivo, y coadyuve a terminar con el conflicto interno que actualmente afecta a toda la población de dicho Estado. Ante esta situación, las instancias internacionales deben continuar con su rol de facilitadoras para que se llegue a un punto de acuerdo que permita terminar con la crisis que se vive actualmente en el Estado de Siria. 


\section{Referencias bibliográficas}

Baruch Pnina, Sharvit \& Weinstock, Brandon, "Military intervention in Syria: is it legal?", INSS Insights, 2013, (465), Institute for National Security Studies, Tel Aviv.

Ertuğrul, Doğan, "A test for 'Turkey’s foreign policy: the Syria crisis”, Tesev's Foreign Policy Bulletin, 2012, The Turkish Economic and Social Studies Foundation, Estambul.

Miller, Vaughne, us Government and Congress Response to Syria 8 (House of Commons, Standard Note: SNIA 6721), 2013.

Nikitin, Mary Beth (coord.), Syria's chemical weapons: issues for Congress, Congressional Research Service, Working Paper R-43201, 2013.

Sasnal, Patrycja, "The onset of civil war in Syria", Bulletin, 2012, 339, (6), Polish Institute of International Affairs, Varsovia.

\section{Recursos de internet}

Agencia Reuters, “China's Xi tells Obama Syria crisis can't be resolved with military strike", in http://www.reuters.com/article/2013/09/06/ us-syria-crisis-china-idUSBRE9850BY20130906, consulta de octubre 18 de 2013.

Centro de Noticias de la ONU, "Siria: Brahimi advierte que cualquier acción militar debe ser autorizada por la ONU", en http:/ / www.un.org/spanish/News/story.asp?NewsID=27309\#.U11xwRAuekc, consulta de octubre 10 de 2013.

Departamento de Estado, "A framework for the elimination of Syrian chemical weapons", en http://blogs.state.gov/stories/2013/09/15/ framework-elimination-syrian-chemical-weapons, consulta de octubre 2 de 2013.

IIP Digital, "Declaración del Secretario de Estado de los Estados Unidos sobre la situación de Siria", en http://iipdigital.usembassy.gov/st/ spanish/texttrans/2013/08/20130830282055.html\#axzz2gfvhHzlO, consulta de octubre 7 de 2013.

IIP Digital, "La Casa Blanca se refiere a evaluación del uso de armas químicas por Siria”, en http://iipdigital.usembassy.gov/st/spanish/texttrans/2013/08/20130830282038.html\#axzz2gfvhHzlO, consulta de octubre 3 de 2013.

Organización para la Prohibición de las Armas Químicas, "Estados miembros de la Organización para la Prohibición de las Armas Químicas 
(OPAQ)", en http://www.opcw.org/nc/sp/acerca-de-la-opaq/estados-miembros/?tx_opcwmemberstate_pi1 [sortField] $=0 \& t x \_o p c w-$ memberstate_pi1[sortReverse] $=0 \&$ tx_opcwmemberstate_pi1[ASP_ PAGE] $=2 \&$ tx_opcwmemberstate_pi1 [pointer] $=0$, consulta de octubre $1^{\circ}$ de 2013.

Organización para la Prohibición de las Armas Químicas/News, "UN Secretary-General, OPCW Director-General Meet in The Hague", en http://www.opcw.org/sp/news/article/un-secretary-generalopcw-director-general-meet-in-the-hague/, consulta de octubre 16 de 2013.

Radio La Voz Rusa, "Putin: Rusia podría realizar una intervención militar en Siria", en http://spanish.ruvr.ru/news/2013_09_04/Putin-admitela-posibilidad-de-acuerdo-de-Rusia-con-una-operaci-n-militar-enSiria-9071/, consulta de octubre 12 de 2013.

The White House, "Statemente by the Presiden ton Syria", en http:/ /www. whitehouse.gov/the-press-office/2013/08/31/statement-presidentsyria, consulta de octubre 12 de 2013.

Zeit Online, "Syriens Giftgasbestände sollen größtenteils nicht waffenfähig sein", en http://www.zeit.de/politik/ausland/2013-09/syrien-giftgas-fluessigkeit, consulta de octubre 28 de 2013.

\section{Tratados Internacionales}

Carta de las Naciones Unidas.

Convención sobre la Prohibición del Desarrollo, la Producción, el Almacenamiento y el Empleo de Armas Químicas y sobre su Destrucción. 13 de enero de 1993.

Protocolo de Ginebra sobre Prohibición del Empleo en la Guerra de Gases Asfixiantes, Tóxicos o Similares o Procedimientos Análogos. 17 de junio de 1925.

\section{Documentos oficiales}

\section{Documentos de la Organización de las Naciones Unidas}

Reglamento del Consejo de Seguridad.

un, Secretaría General, Informe de la misión de las Naciones Unidas encargada de investigar las denuncias relativas al empleo de armas químicas en la República Árabe Siria acerca del incidente ocurrido en la zona de Ghouta, en Damasco, el 
21 de agosto de 2013, Doc. A/67/997, S/2013/553 (16 de septiembre de 2013).

UNGA, Resolución 2625 (xxv), Declaración sobre los Principios de Derecbo Internacional Referentes a las Relaciones de Amistad y a la Cooperación entre los Estados de Conformidad con la Carta de las Naciones Unidas, Resolución de la Asamblea General (24 de octubre de 1970).

UNGA, Resolución 55/283, Anexo, Acuerdo de Relación entre las Naciones Unidas y la Organización para la Probibición de las Armas Quimicas (24 de septiembre de 2001).

unsC, Carta de fecha 24 de enero de 2012 dirigida al Presidente del Consejo de Seguridad por el Secretario General, Doc. S/2012/71 (30 de enero de 2012).

UNSC, Documento del Consejo de Seguridad, Informe del Secretario General relativo a la aplicación de la Resolución 2043 (2012), Doc. S/2012/523 (6 de julio de 2012).

unsC, Nota del Presidente del Consejo de Seguridad, Doc. S/23500 (21 de enero 21 de 1992).

UNSC, Resolución 1540 (2004).

UNSC, Resolución 2118 (2013).

unsC, Resolución 2118 (2013).

UNSC, Resolución 620 (1988).

unsC, Sesión 6710ª , Doc. S/PV.6710 (31 de enero de 2012).

unsC, Sesión 7038a , Doc. S/PV. 7038 (27 de septiembre de 2013).

\section{Documentos de la Organización} para la Prohibición de las Armas Químicas

OPAQ, Doc. EC-M-33/DEC.1.

OPAQ, Doc. EC-M-33/NAT.1, Anexo A.

OPAQ, Doc. EC-M-33/NAT.1, Anexo.

\section{Documentos del Senado de los Estados Unidos de América}

S.J., Res. 21, Authorization for the use of military force against the government of Syria to respond to use of chemical weapons. 